\title{
The positiveness of lower limits of the Hoffman constant in parametric polyhedral programs
}

\author{
A. Jourani · D. Zagrodny
}

Received: 16 September 2010 / Accepted: 17 May 2011 / Published online: 31 May 2011

(C) The Author(s) 2011. This article is published with open access at Springerlink.com

\begin{abstract}
If $K(t)$ are sets of admissible solutions in parametric programs then it is natural to ask about the Lipschitz-like property and the lower semi-continuity of the multifunction. Answers to this question are related to the problem of the continuity or Lipschitz continuity of the value function, namely having the lower semi-continuity of $K(\cdot)$ we get the upper semi-continuity of the function easily and the Lipschitz-like property of $K(\cdot)$ leads to the Lipschitz-continuity of it. Herein sufficient conditions to get these properties of the polyhedral multifunction of admissible solutions are given in terms of the lower limit of the Hoffman constant. It is shown that the multifunction is Lipschitz-like at these parameters at which the lower limit of the Hoffman constant are positive.
\end{abstract}

Keywords Parametric programming $\cdot$ Hoffman constant $\cdot$ Error bound · Mosco convergence $\cdot$ Attouch's theorem - Convex functions - Subdifferentials · Polyhedrals

Mathematics Subject Classification (2000) $\quad 49 \mathrm{~J} 40$

This paper was written while the second author was visiting Professor at the University of Burgundy. The author would like to thank the authority of the University for the hospitality.

A. Jourani

Université de Bourgogne, UFR Sciences et Techniques, Institut de Mathématiques de Bourgogne, UMR 5584 CNRS, BP 47 870, 21078 Dijon Cedex, France

e-mail: jourani@u-bourgogne.fr

D. Zagrodny

Faculty of Mathematics and Natural Science, College of Science, Cardinal Stefan Wyszyński University, Dewajtis 5, 01-815 Warsaw, Poland

D. Zagrodny $(\varangle)$

System Research Institute, Polish Academy of Sciences, ul. Newelska 6, 01-447 Warsaw, Poland

e-mail: d.zagrodny@uksw.edu.pl 


\section{Introduction}

Let $(X,\|\cdot\|)$ be a real Banach space and $X^{*}$ be its topological dual. For a set $I$ of indices, a metric space $\left(T, d_{T}\right)$ and mappings $a_{i}^{*}: T \mapsto X^{*}$ and $b_{i}: T \mapsto \mathbf{R}, i \in I$, we study the following inequality system

$$
\left\langle a_{i}^{*}(t), x\right\rangle+b_{i}(t) \leq 0 \quad \text { for all } i \in I,
$$

where $\langle\cdot, \cdot\rangle$ refers to the pairing between $X$ and $X^{*}$. This system is viewed as depending on the parameter $t \in T$, so for each $t \in T$, let $K(t)$ be the (possibly empty) set of solutions to (1) with respect to $x$. We are interested in a local behavior of the multifunction $K: T \rightrightarrows X$ around a fixed element $t_{0} \in T$. Our attention is mainly focused on conditions under which $K$ is Lipschitz-like at $t_{0}$, in the sense that there exist a neighborhood $U_{0}$ of $t_{0}$ and $\gamma>0$ such that

$$
K\left(t^{\prime}\right) \cap \mathbf{B}(0, r) \subset K(t)+\gamma(r+1) d_{T}\left(t, t^{\prime}\right) \mathbf{B}(0,1), \quad \forall t, t^{\prime} \in U_{0}, \forall r>0 .
$$

or $K$ is lower semi-continuous at $t_{0}$, i.e.

$$
K\left(t_{0}\right) \subset \liminf _{t \rightarrow t_{0}} K(t),
$$

where $\mathbf{B}(0, r)$ denotes the ball at 0 with radius $r$ in $X$ and $\lim \inf _{t \rightarrow t_{0}}$ stands for the lower limit of sets. These properties play a central role in parametric programming, we refer to [5] for several facts on the continuity of $K(\cdot)$. They allow to investigate the behavior and the properties of solution sets of optimization problems under variations of the describing parameters. To be more precise, let us consider the following problem

$$
\min _{x \in K(t)} g(t, x),
$$

where $g: T \times X \mapsto \mathbf{R}$ is a given function, which is assumed to be convex in the second variable. Changing $t$ over $T$, we will get a family of problems $\left(P_{t}\right)$ whose values and sets of solutions are given, respectively, by

$$
\begin{aligned}
& v(t)=\inf _{x \in K(t)} g(t, x) \\
& S(t)=\left\{y \in K(t): \quad g(t, y)=\min _{x \in K(t)} g(t, x)\right\},
\end{aligned}
$$

where $K(t):=\{x \in X: g(t, x) \leq 0\}$. The obtained function $v$ is called value or marginal or cost function. The behavior of it is related to that of the solution sets $S(t)$. In order to observe it let us indicate some links among $K, S$ and $v$. For this reason fix $t_{0} \in T$ and suppose that $g, a_{i}^{*}, b_{i}$ are continuous. It is easy to observe that the following implication

$$
\begin{aligned}
& \text { lower semi-continuity of } \mathrm{K} \text { at } t_{0} \\
& \quad \text { and } z_{n} \longrightarrow z_{0}, t_{n} \longrightarrow t_{0}, z_{n} \in S\left(t_{n}\right) \forall n \in \mathbb{N} \Longrightarrow z_{0} \in S\left(t_{0}\right)
\end{aligned}
$$

holds true, see also [5, Theorems 4.2.1 and 4.2.2]. Additionally, imposing a uniform compactness assumption on $S(t)$ around $t_{0}$ and some continuity properties on $g, a_{i}^{*}, b_{i}$, we have lower semi-continuity of $v$ at $t_{0}$ as well as the following equivalence

$$
\text { upper semicontinuity of } S \text { at } t_{0} \Longleftrightarrow \text { upper semicontinuity of } v \text { at } t_{0}
$$

holds true. This fact is commonly known see [11, Theorem 5] or [6, Proposition 12], we refer also to [18]. Of course to get $v$ more smooth we have to assume more on involved functions, we refer to $[20,21]$ and the references therein for several facts on that. 
We see that the lower semi-continuity of $K(\cdot)$ is essential to get the upper semi-continuity of $v$. Herein we provide it using the Hoffman constant. This constant is given by

$$
\alpha_{f}(t):=\inf _{x \notin K(t)} \frac{f(t, x)}{d(x, K(t))},
$$

where $f(t, x)=\sup _{i \in I}\left[\left\langle a_{i}^{*}(t), x\right\rangle+b_{i}(t)\right], K(t):=\{x \in X: f(t, x) \leq 0\}$ see [10], and $\inf \emptyset:=\infty$. Many authors have presented and studied explicit representations of Hoffman constants, we refer to $[3,4,6,7,10,14-17,23,28,29]$ and references therein, see also [19]. In [7, Theorem 5.1] (under the assumption $K(t) \neq \emptyset$ ) it is shown that

$$
c \leq \alpha_{f}(t) \Longleftrightarrow c \leq \inf _{x \notin K(t)} d\left(0, \partial_{x} f(t, x)\right)
$$

where $\partial_{x} f(t, x)$ is the Fenchel subdifferential of the convex function $x \mapsto f(t, x)$ and $d\left(0, \partial_{x} f(t, x)\right)$ is the distance between 0 and $\partial_{x} f(t, x)$ with respect to the norm of $X^{*}$. Relation (8) is equivalent to the following one

$$
\alpha_{f}(t)=\inf _{x \notin K(t)} d\left(0, \partial_{x} f(t, x)\right)
$$

obtained in [4]. This representation of the Hoffman constant allows us to use a subddifferential calculus to show that the inequality

$$
\liminf _{t \rightarrow t_{0}} \alpha_{f}(t)>0
$$

entails the lower semi-continuity of $K(\cdot)$, see Theorem 5.1, or the Lipschitz continuity, see Theorem 5.2, thus (2) and (3) hold. Unfortunately, the function $t \mapsto \alpha_{f}(t)$ is not lower semi-continuous even in simple cases, as it is shown in Sect. 3. It means that it is not enough to impose conditions preserving that $\alpha_{f}\left(t_{0}\right)>0$ to get the positiveness of the lower limit at $t_{0}$. The problem is much more complicated. In Sect. 4 we present conditions implying the positiveness whenever $I$ is finite or denumerable. The case $I$ is denumerable involves the Attouch technique of approximation of subgradients by "better" ones in getting the inequality, see Theorems 4.8 and 4.14. This technique can be used only in reflexive Banach spaces or more generally in weakly compactly generated Banach spaces. We do not know how to get this results in general Banach spaces, this is an open problem. Whenever $I$ is finite or $f$ can be expressed as the maximum of a finite number of affine functions, see [24] for some information on this technique, it is easier to evaluate the subdifferential $\partial_{x} f(t, x)$, so (9) can be applied to get the inequality $\lim _{\inf } t_{t \rightarrow t_{0}} \alpha_{f}(t)>0$, see Example 4.2 and Proposition 4.5. Let us also mention that whenever $I$ is a finite set, $K(t) \neq \emptyset$ near $t_{0}$, functionals $a_{i}^{*}$ do not depend on $t$ and at least one of them is different from zero, then $\lim \inf _{t \rightarrow t_{0}} \alpha_{f}(t)>0$, see Proposition 4.6 and Corollary 4.7 for details.

When we compare Proposition 4.5, Theorems 4.8 and 4.14 it turns out that they are of different nature. We present them in those miscellaneous forms in order to point out that there are several possibilities to preserve the positiveness of the limit $\lim _{\inf } \operatorname{lit}_{0} \alpha_{f}(t)>0$ by examples. Of course there are possibilities to produce theorems like Proposition 4.5 in the reflexive or weakly compactly generated Banach space set up with denumerable families of affine mappings, and the reverse is also possible.

Finally we would like to thank the referee for his remarks, which eliminated some gaps in the presentation. 


\section{Properties of subgradients of convex functions}

In this section several properties of lower semi-continuous functions defined on a real Banach space are recalled, we refer to [22] for the definition of lower semi-continuous proper convex function and their properties. When $X$ is a Banach space then the weak topology is denoted by $\sigma\left(X, X^{*}\right)$, the weak* topology by $\sigma\left(X^{*}, X\right)$, we refer to [12] for the definitions of the weak topologies, weak convergence, weak* convergence and for the definition of the reflexive Banach space. The closed and the open unit balls of $X$ are denoted by $\mathbf{B}$ and $\stackrel{\circ}{B}$.

The (Fenchel) subdifferential of a convex function $f: X \mapsto \mathbb{R} \cup\{\infty\}$ at a point $x$ is the subset of the dual space $X^{*}$ given by

$$
\partial f(x)=\left\{x^{*} \in X^{*}:\left\langle x^{*}, u-x\right\rangle \leq f(u)-f(x) \forall u \in X\right\}
$$

if $x$ is a point of the domain of $f$, where $\operatorname{dom} f:=\{u \in X: f(u)<\infty\}$, and the subdifferential is the emptyset otherwise.

It follows from this definition that for every $\varepsilon>0$ the following assertions are equivalent

$$
\begin{aligned}
\mathbf{B}(0, \varepsilon) & \subset \partial f(x), \\
\forall u \in X, \varepsilon\|u-x\| & \leq f(u)-f(x) .
\end{aligned}
$$

Either (10) or (11) ensures that $x$ is an isolated minimum of $f$.

Below we recall two results allowing us to approximate a subgradient of a convex function by subgradients of convex functions, which subgradients are easier to calculate. For this reason let us recall the notion of the Mosco convergence. In doing this we follow [2], we refer also to [1] for more information on the Mosco convergence.

Definition 2.1 Let $X$ be a Banach space and $f, f_{n}: X \longrightarrow \mathbb{R} \cup\{\infty\}$ for every $n \in \mathbb{N}$. We say that $f=$ Mosco $-\lim _{n \rightarrow \infty} f_{n}$ if the two following conditions are satisfied:

(S1) whenever $\left\{x_{n}\right\}_{n=1}^{\infty}$ is a sequence weakly convergent to $x$, then $f(x) \leq \liminf _{n} \longrightarrow \infty$ $f_{n}\left(x_{n}\right)$

(S2) for each $x \in X$ there exists a sequence $\left\{x_{n}\right\}_{n=1}^{\infty}$ converging in norm to $x$ for which $f(x)=\lim _{n \rightarrow \infty} f_{n}\left(x_{n}\right)$.

It is not difficult to notice that if $\left\{f_{n}\right\}_{n=1}^{\infty}$ is a nondecreasing sequence of lower semicontinuous convex functions and for every $x \in X$

$$
f(x):=\lim _{n \longrightarrow \infty} f_{n}(x),
$$

then (S1) and (S2) are satisfied.

First result concerning the approximations of subgradients by better ones is a consequence of the Attouch theorem (the necessity part), see [1].

Theorem 2.2 Let $f, f_{1}, f_{2}, \ldots E \longrightarrow \mathbb{R} \cup\{+\infty\}$ be convex lower semi-continuos functions on a reflexive Banach space $X$ and $f=$ Mosco $-\lim _{n \rightarrow \infty} f_{n}$. For any $x^{*} \in \partial f(x)$ there are sequences $\left\{x_{n}\right\} \subset X,\left\{x_{n}^{*}\right\} \subset X^{*}$ such that

a: $\lim _{n \rightarrow \infty} x_{n}=x, \lim _{n \rightarrow \infty} f_{n}\left(x_{n}\right)=f(x)$;

$\mathrm{b}: \quad x_{n}^{*} \in \partial f_{n}\left(x_{n}\right)$ for every $n \in \mathbb{N}$ and $x^{*}=\lim _{n \longrightarrow \infty} x_{n}^{*}$.

We recall that a Banach space is WCG (weakly compactly generated) if there exists a weakly compact subset $W$ of $X$ that spans a dense linear space in $X$, one can always assume that $W$ is convex, we refer to $[8,22]$ for detailed information on WCG spaces. Below 
we recall, see [25], that if $X$ is a weakly compactly generated Banach space and $f=$ Mosco $-\lim _{n \rightarrow \infty} f_{n}$, then for every $\left(x, x^{*}\right) \in \partial f$ there is a sequence $\left\{\left(x_{n}, x_{n}^{*}\right)\right\}_{n=1}^{\infty}$ such that $\left(x_{n}, x_{n}^{*}\right) \in \partial f_{n}, x_{n} \longrightarrow x, f_{n}\left(x_{n}\right) \longrightarrow f(x)$ and $\lim _{n} \longrightarrow \infty\left\langle x_{n}^{*}, h\right\rangle=\left\langle x^{*}, h\right\rangle$ for every $h \in X$, we refer to [25-27] for more.

Theorem 2.3 Let $X$ be a WCG Banach space, $\left(x, x^{*}\right) \in X \times X^{*}$ be fixed and $f: X \longrightarrow$ $\mathbb{R} \cup\{+\infty\}$ be a lower semi-continuous convex function such that $f(x) \in \mathbb{R}, x^{*} \in \partial f(x)$. Assume that $f_{n}: X \longrightarrow \mathbb{R} \cup\{+\infty\}$ are lower semi-continuous convex functions such that:

i: $\quad f=$ Mosco $-\lim _{n \rightarrow \infty} f_{n}$;

ii: there is an open nonempty subset $U$ of $X$ and a constant $c \in \mathbb{R}$ such that for every $u \in U$ and $n \in \mathbb{N}$ we have $f_{n}(u) \leq c$.

Then there are sequences $\left\{x_{n}\right\}_{n=1}^{\infty} \subset E$ and $\left\{x_{n}^{*}\right\}_{n=1}^{\infty} \subset X^{*}$ such that:

iii: $\lim _{n \rightarrow \infty} x_{n}=x, \lim _{n \rightarrow \infty} f_{n}\left(x_{n}\right)=f(x)$;

iv: $\forall n \in \mathbb{N}, \quad x_{n}^{*} \in \partial f_{n}\left(x_{n}\right)$;

v: $\forall h \in X, \quad \lim _{n \longrightarrow \infty}\left\langle x_{n}^{*}, h\right\rangle=\left\langle x^{*}, h\right\rangle$.

Finally let us recall the Ekeland variational principle, see $[9,22]$ for more.

Theorem 2.4 (Ekeland Variational Principle) Assume that $f: X \longrightarrow \mathbb{R} \cup\{\infty\}$ is a lower semi-continuous function on a Banach space $X$, bounded from below. For any $\epsilon>0, \lambda>0$, $x_{0} \in E$ such that $f\left(x_{0}\right) \leq \inf _{X} f+\epsilon \lambda$ there is a point $z \in X$ satisfying

$$
\left\|z-x_{0}\right\| \leq \lambda, f(z) \leq f\left(x_{0}\right)
$$

and

$$
\epsilon\|z-x\|+f(x)>f(z) \text { for every } x \neq z \text {. }
$$

\section{Examples of lower semi-continuity of admissible sets with the lack of lower semi-continuity of the error bounds}

In this section three simple examples of parametric convex programs are presented, where the sets of the admissible solutions are lower semi-continuous with respect to parameter but the Hoffman constants are not lower semi-continuous. Thus the lower semi-continuity of the multifunction of admissible solutions may not be linked to the lower semi-continuity of the error bound function.

Example 3.1 Let us put $T:=[0,1]$ and for every $t \in T$

$$
\begin{array}{r}
a_{1}(t):=0, a_{2}(t):=1+t, a_{3}(t):=2 \\
b_{1}(t):=0, b_{2}(t):=0, b_{3}(t):=t^{2}-t,
\end{array}
$$

and for every $x \in \mathbb{R}$

$$
f(t, x):=\max _{i \in\{1,2,3\}} a_{i}(t) x+b_{i}(t) .
$$

Let us observe that $f$ is Lipschitz continuous on $T \times \mathbb{R}$ and for every $t$ the function $f(t, \cdot)$ is convex, moreover

$$
f(t, x)= \begin{cases}0, & \text { if } x \leq 0 \\ (1+t) x, & \text { if } 0<x \leq t \\ 2 x+t^{2}-t, & \text { if } t<x\end{cases}
$$


For every $t \in T$ denote

$$
\alpha_{f}(t):=\inf _{y \in \mathbb{R}, f(t, y)>0} d\left(0, \partial_{x} f(t, y)\right),
$$

where $\partial_{x} f(t, y)$ stands for the subdifferential of $f$ with respect to the second variable at $y$, and

$$
K_{f}(t):=\{x \in \mathbb{R} \mid f(t, x) \leq 0\} .
$$

For every $t \in] 0,1\left[\right.$ we have $\alpha_{f}(t)=1+t, \alpha_{f}(0)=2$ and

$$
\liminf _{t \searrow 0} \alpha_{f}(t)=1 \text {. }
$$

Thus $\alpha_{f}(\cdot)$ is not lower semi-continuous at 0 but $\left.K_{f}(t)=\right]-\infty, 0$ ] for every $t \in T$ and $K_{f}(\cdot)$ is lower semi-continuous on $T$.

Below we provide another example, where the same phenomena occurs but the lower limit in (12) is equal to 0 , hence we infer that the positiveness of the lower limit of the Hoffman constants is not necessary for the lower semi-continuity of the admissible sets of solutions. The second example is a slight modification of the first one, namely

Example 3.2

$$
f(t, x)= \begin{cases}0, & \text { if } x \leq 0 \\ t x, & \text { if } 0<x \leq t \\ x+t^{2}-t, & \text { if } t<x\end{cases}
$$

For every $t \in T$ put

$$
\alpha_{f}(t):=\inf _{y \in \mathbb{R}, f(t, y)>0} d\left(0, \partial_{x} f(t, y)\right)
$$

and

$$
K_{f}(t):=\{x \in \mathbb{R} \mid f(t, x) \leq 0\} .
$$

For every $t \in] 0,1\left[\right.$ we have $\alpha_{f}(t)=t, \alpha_{f}(0)=1$ and

$$
\liminf _{t \searrow 0} \alpha_{f}(t)=0 .
$$

Again $\alpha_{f}(\cdot)$ is not lower semi-continuous at 0 but $\left.K_{f}(t)=\right]-\infty, 0$ ] for every $t \in T$, so $K_{f}(\cdot)$ is lower semi-continuous on $T$.

The third example is just to show that even assuming that

$$
0 \notin \text { bd conv }\left\{a_{i}^{*}\left(t_{0}\right) \mid i \in J\right\} \text { for every subset } J \subset\{1,2,3\}
$$

we do not have the lower semi-continuity of the error bounds, see also (19) in Remark 4.4

Example 3.3

$$
f(t, x)= \begin{cases}\frac{x}{2}, & \text { if } x \leq 0 \\ (1+t) x, & \text { if } 0<x \leq t \\ 2 x+t^{2}-t, & \text { if } t<x\end{cases}
$$

For every $t \in T$ put

$$
\alpha_{f}(t):=\inf _{y \in \mathbb{R}, f(t, y)>0} d\left(0, \partial_{x} f(t, y)\right)
$$


and

$$
K_{f}(t):=\{x \in \mathbb{R} \mid f(t, x) \leq 0\} .
$$

For every $t \in] 0,1\left[\right.$ we have $\alpha_{f}(t)=(1+t), \alpha_{f}(0)=2$ and

$$
\liminf _{t \searrow 0} \alpha_{f}(t)=1 \text {. }
$$

Again $\alpha_{f}(\cdot)$ is not lower semi-continuous at 0 but

$$
0 \notin \text { bd conv }\left\{a_{i}^{*}(0) \mid i \in J\right\} \text { for every subset } J \subset\{1,2,3\} .
$$

Of course $K_{f}(t)=$ ] $\left.-\infty, 0\right]$ for every $t \in T$, so $K_{f}(\cdot)$ is lower semi-continuous on $T$.

Let us observe that all the examples can be rearranged to have $f(t, \cdot)$ coercive, so the admissible sets would be bounded. For this reason it is enough to add $a_{4}(t) \equiv b_{4} \equiv-1$ and in the definition of $f$ take the maximum from the four affine functions instead of the three, see Example 3.1.

\section{The positiveness of the lower limits of the Hoffman constants}

Throughout the paper let $X$ be a real Banach space, $T$ be a metric space, $I$ be a nonempty set of indices and the family of mappings $\left\{\left(a_{i}^{*}, b_{i}\right)\right\}$ be given, where

$$
a_{i}^{*}: T \longrightarrow X^{*}, \quad b_{i}: T \longrightarrow \mathbb{R} \text {. }
$$

Let us define

$$
f(t, x):=\sup \left\{\left\langle a_{i}^{*}(t), x\right\rangle+b_{i}(t) \mid i \in I\right\},
$$

and for every $\epsilon \geq 0$

$$
I(t, x, \epsilon):=\left\{i \in I \mid\left\langle a_{i}^{*}(t), x\right\rangle+b_{i}(t)+\epsilon \geq f(t, x)\right\}
$$

and

$$
\alpha_{f}(t):=\inf _{y \in X, f(t, y)>0} d\left(0, \partial_{x} f(t, y)\right)
$$

and

$$
K_{f}(t):=\{x \in X \mid f(t, x) \leq 0\},
$$

where the infimum over the empty set is $+\infty$.

We start with a simple observation that having 0 inside the interior of the subdifferential we get that the lower limit of the error bounds is positive, namely

Proposition 4.1 Fix $\left(t_{0}, x_{0}\right) \in T \times X$ and let us assume that $f: T \times X \longrightarrow \mathbb{R} \cup\{+\infty\}$ is such that $f\left(t_{0}, x_{0}\right)=0$ and for some $\epsilon>0$ and every $t \in T$ in some neighborhood of $t_{0}$, say for every $t \in U\left(t_{0}\right)$, we have

$$
\mathbf{B}(0, \epsilon) \subset \partial_{x} f\left(t, x_{0}\right),
$$

and $K_{f}(t) \neq \emptyset$, then for every $t \in U\left(t_{0}\right)$ we have $\alpha_{f}(t) \geq \epsilon$, thus

$$
\liminf _{t \longrightarrow t_{0}} \alpha_{f}(t) \geq \epsilon \text {. }
$$


Proof For every $x \in X$ and every $t \in U\left(t_{0}\right)$ by the equivalence (10) $\Longleftrightarrow$ (11) we have

$$
\epsilon\left\|x-x_{0}\right\| \leq f(t, x)-f\left(t, x_{0}\right) .
$$

Thus if $x^{*} \in \partial_{x} f(t, x)$, then

$$
\epsilon\left\|x-x_{0}\right\| \leq f(t, x)-f\left(t, x_{0}\right) \leq\left\langle x^{*}, x-x_{0}\right\rangle,
$$

which implies $\left\|x^{*}\right\| \geq \epsilon$ for every $x \in X \backslash\left\{x_{0}\right\}$ and $x^{*} \in \partial_{x} f(t, x)$. In order to complete the proof let us observe that $f\left(t, x_{0}\right) \leq 0$ for every $t \in U\left(t_{0}\right)$. In fact, by (16) we have $f\left(t, x_{0}\right) \leq f(t, x)$ for every $x \in K_{f}(t)$, so $f\left(t, x_{0}\right) \leq 0$ and by the definition of $\alpha_{f}(\cdot)$ only $x \in X \backslash\left\{x_{0}\right\}$ can be considered when the values of the function are calculated, but then $\left\|x^{*}\right\| \geq \epsilon$ if $x^{*} \in \partial_{x} f(t, x)$, thus $\alpha_{f}(t)=\inf _{y \in X, f(t, y)>0} d\left(0, \partial_{x} f(t, y)\right) \geq \epsilon$ ( keep in mind that the infimum over the empty set is $+\infty)$.

Below we provide an example showing that whenever 0 is in the interior of the polyhedron generated by a finite family $\left\{a_{i}^{*} \mid i \in I\left(t_{0}, x_{0}, 0\right)\right\}$, then (15) is satisfied with $x_{0}=0$

Example 4.2 Assume that for given $\delta>0, \varepsilon_{0}>0$ and $t_{0} \in T$, we have $0 \in K_{f}\left(t_{0}\right)$, the sets $K_{f}(t)$ are nonempty for every $t \in T$ close to $t_{0}$, the mappings $\left\{a_{i}^{*}(\cdot) \mid i \in I\left(t_{0}, 0, \varepsilon_{0}\right)\right\}$ are equi-continuous at $t_{0}$, the mappings $\left\{b_{i}(\cdot) \mid i \in I\left(t, 0, \varepsilon_{0}\right)\right\}$ do not depend on $t$, i.e. $b_{i}(\cdot) \equiv b_{i}$ for every $\left.i \in I\left(t, 0, \varepsilon_{0}\right)\right\}$, and

$$
\mathbf{B}(0,2 \delta) \subset \bigcap_{\varepsilon \in] 0, \varepsilon_{0}[} \mathrm{cl}^{*} \operatorname{conv}\left\{a_{i}^{*}\left(t_{0}\right) \mid i \in I\left(t_{0}, 0, \varepsilon\right)\right\},
$$

then the assumptions of the above proposition are satisfied, where $f$ is defined in (14) and $\mathrm{cl}^{*}$ stands for the closure with respect to the weak* topology.

Indeed, because of the equi-continuity of $\left(a_{i}^{*}(\cdot)\right)$, there exists a neighborhood $U\left(t_{0}\right)$ of $t_{0}$ such that

$$
\left\|a_{i}^{*}(t)-a_{i}^{*}\left(t_{0}\right)\right\| \leq \delta \forall t \in U\left(t_{0}\right) \text { and } i \in I\left(t_{0}, 0, \varepsilon_{0}\right) .
$$

Fix $x \in X, t \in T$ such that $K_{f}(t) \neq \emptyset$ and let $\left.\varepsilon \in\right] 0, \varepsilon_{0}\left[\right.$ be arbitrary. For each $z^{*} \in$ conv $\left\{a_{i}^{*}\left(t_{0}\right) \mid i \in I\left(t_{0}, 0, \varepsilon\right)\right\}$, there exist a finite subset $J \subset I\left(t_{0}, 0, \varepsilon\right)$ and non-negative numbers $\left(\lambda_{i}\right)_{i \in J}, \sum_{i \in J} \lambda_{i}=1$, such that $z^{*}=\sum_{i \in J} \lambda_{i} a_{i}^{*}\left(t_{0}\right)$. Then

$$
\begin{aligned}
f(t, x)-f\left(t_{0}, 0\right) & \geq \sum_{i \in J} \lambda_{i}\left[\left\langle a_{i}^{*}(t), x\right\rangle+b_{i}\right]-\sum_{i \in J} \lambda_{i}\left[\left\langle a_{i}^{*}\left(t_{0}\right), 0\right\rangle+b_{i}\right]-\varepsilon \\
& =\sum_{i \in J}\left[\lambda_{i}\left\langle a_{i}^{*}(t)-a_{i}^{*}\left(t_{0}\right), x\right\rangle\right]+\sum_{i \in J} \lambda_{i}\left\langle a_{i}^{*}\left(t_{0}\right), x\right\rangle-\varepsilon \\
& \geq-\sum_{i \in J} \lambda_{i}\left\|a_{i}^{*}(t)-a_{i}^{*}\left(t_{0}\right)\right\|\|x\|+\left\langle z^{*}, x\right\rangle-\varepsilon \\
& \geq-\delta\|x\|+\left\langle z^{*}, x\right\rangle-\varepsilon .
\end{aligned}
$$

Hence for each $z^{*} \in \mathrm{cl}^{*} \operatorname{conv}\left\{a_{i}^{*}\left(t_{0}\right) \mid i \in I\left(t_{0}, 0, \varepsilon\right)\right\}$

$$
f(t, x)-f\left(t_{0}, 0\right) \geq-\delta\|x\|+\left\langle z^{*}, x\right\rangle-\varepsilon .
$$

The last inequality is also true for all $z^{*} \in \mathbf{B}(0,2 \delta)$ and hence $f(t, x)-f\left(t_{0}, 0\right) \geq \delta\|x\|-\varepsilon$. Since $\varepsilon$ is arbitrary in $] 0, \varepsilon_{0}[$, we have

$$
f(t, x)-f\left(t_{0}, 0\right) \geq \delta\|x\| \quad \forall x \in X .
$$


Using the assumption that the mappings $\left\{b_{i}(\cdot) \mid i \in I\left(t, 0, \varepsilon_{0}\right)\right\}$ do not depend on $t$, we have $f\left(t_{0}, 0\right)=f(t, 0)$ for all $t$, and then

$$
f(t, x)-f(t, 0) \geq \delta\|x\| \quad \forall x \in X \quad \forall t \in U\left(t_{0}\right)
$$

or equivalently

$$
\mathbf{B}(0, \delta) \subset \partial_{x} f(t, 0) \forall t \in U\left(t_{0}\right)
$$

and this is exactly relation (15).

Remark 4.3 The example above holds true if we replace $I\left(t, 0, \varepsilon_{0}\right)$ by $I(t, 0,0)$ and condition (17) by the following one

$$
\mathbf{B}(0,2 \delta) \subset \mathrm{cl}^{*} \operatorname{conv}\left\{a_{i}^{*}\left(t_{0}\right) \mid i \in I\left(t_{0}, 0,0\right)\right\},
$$

Remark 4.4 If the space $X$ is a finite dimensional, $I$ is finite, the mappings $\left\{a_{i}^{*}(\cdot) \mid i \in I\right.$ $\left.\left(t_{0}, 0,0\right)\right\}$ are continuous at $t_{0}$, then (17) implies (15).

It is also easy to observe that assuming, similarly to [4], that $I$ is finite and for every subset $J \subset I\left(t_{0}, x_{0}, 0\right)$

$$
0 \notin \mathrm{bd} \operatorname{conv}\left\{a_{i}^{*}\left(t_{0}\right) \mid i \in J\right\}
$$

either (17) holds true or it does not hold but then (19) ensures

$$
\stackrel{\circ}{B}(0, \delta) \cap \bigcup_{t \in U\left(t_{0}\right)} \operatorname{conv}\left\{a_{i}^{*}(t) \mid i \in I\left(t, x_{0}, 0\right)\right\}=\emptyset
$$

for some $\delta>0$ and a neighborhood of $t_{0}$, say $U\left(t_{0}\right)$.

It is natural to ask what happens if (15) does not hold. Below we give partial answers to this question whenever the set $I$ is either finite or denumerable. In the Proposition below we assume only that sets of almost active constraints are finite.

Proposition 4.5 Let us fix $t_{0} \in T$ and assume that for some $\epsilon>0$ and a neighborhood of $t_{0}$, say $U\left(t_{0}\right) \subset T$, the sets

$$
\bigcup_{y \notin K_{f}(t)} I(t, y, \epsilon)
$$

are nonempty and finite for every $t \in U\left(t_{0}\right)$. If for some $\delta>0$

$$
\begin{aligned}
& \stackrel{\circ}{B}(0, \delta) \cap \underset{t \in U\left(t_{0}\right), y \in \operatorname{dom} f(t, \cdot) \backslash K_{f}(t), f(t, y)>0}{\bigcup^{\prime}}\left(\operatorname{conv}\left\{a_{i}^{*}(t) \mid i \in I(t, y, \epsilon)\right\}\right. \\
& +N(\operatorname{dom} f(t, \cdot), y))=\emptyset,
\end{aligned}
$$

then $\alpha_{f}(t) \geq \delta$ for every $t \in U\left(t_{0}\right)$, thus

$$
\liminf _{t \rightarrow t_{0}} \alpha_{f}(t) \geq \delta
$$

where $N(\operatorname{dom} f(t, \cdot), y)$ is the normal cone to dom $f(t, \cdot)$ at $y$, i.e.

$N(\operatorname{dom} f(t, \cdot), y):=\left\{x^{*} \in X^{*} \mid\left\langle x^{*}, u-y\right\rangle \leq 0 \forall u \in \operatorname{dom} f(t, \cdot)\right\}$. 
Proof Let us fix $t \in U\left(t_{0}\right)$ and $y \in X$ such that $\left.f(t, y) \in\right] 0, \infty\left[\right.$ and $\partial_{x} f(t, y) \neq \emptyset$. Take $u \in$ $\operatorname{dom} f(t, \cdot)$. By the lower semi-continuity and the convexity of $f(t, \cdot)$ there is $\mu>0$ such that $f(t, y+s(u-y)) \in] 0, \infty\left[\right.$ for every $s \in[0, \mu]$, hence the set $\bigcup_{s \in[0, \mu]} I(t, y+s(u-y), \epsilon)$ is finite. Because of the finiteness of $I(t, y, \epsilon)$ and the continuity of $f(t, \cdot)$ on the segment $[y, u]$ we have $I(t, y+s(u-y), \epsilon) \subset I(t, y, \epsilon)$, for $s>0$ small enough. Indeed, if $I\left(t, y+s_{n}(u-y), \epsilon\right) \backslash I(t, y, \epsilon) \neq \emptyset$ for every $n \in \mathbb{N}$ and some $s_{n} \downarrow 0$, then there is a subsequence $\left(s_{n_{k}}\right)_{k \in \mathbb{N}}$ such that

$$
i_{0} \in I\left(t, y+s_{n_{k}}(u-y), \epsilon\right) \backslash I(t, y, \epsilon)
$$

for some $i_{0} \in \mathbb{N}$ and every $k \in \mathbb{N}$ (keep in mind that $\bigcup_{k=1}^{\infty} I\left(t, y+s_{n_{k}}(u-y), \epsilon\right)$ is finite). Hence

$$
\left\langle a_{i_{0}}^{*}(t), y+s_{n_{k}}(u-y)\right\rangle+b_{i_{0}}(t)+\epsilon \geq f\left(t, y+s_{n_{k}}(u-y)\right) \longrightarrow f(t, y),
$$

so $i_{0} \in I(t, y, \epsilon)$, a contradiction. Thus $I(t, y+s(u-y), \epsilon) \subset I(t, y, \epsilon)$, for $s>0$ small enough. Take any $x^{*} \in \partial_{x} f(t, y)$ and observe that for $\left.s \in\right] 0, \mu$ [ small enough we obtain

$$
\left\langle x^{*}, s(u-y)\right\rangle \leq f(t, y+s(u-y))-f(t, y) \leq \max \left\{\left\langle a_{i}^{*}, s(u-y)\right| i \in I(t, y, \epsilon)\right\} .
$$

Now applying the standard procedure, see for example[12, Theorem p. 87], we get

$$
x^{*} \in \operatorname{conv}\left\{a_{i}^{*}(t) \mid i \in I(t, y, \epsilon)\right\}+N(\operatorname{dom} f(t, \cdot), y),
$$

thus

$$
\partial_{x} f(t, y) \subset \operatorname{conv}\left\{a_{i}^{*}(t) \mid i \in I(t, y, \epsilon)\right\}+N(\operatorname{dom} f(t, \cdot), y) .
$$

Hence by the assumptions for every $t \in U\left(t_{0}\right), y \in X$ with $\infty>f(t, y)>0$ we get (keep in mind that the distance from the empty set is $\infty$ )

$$
d\left(0, \partial_{x} f(t, y)\right) \geq \delta .
$$

Observe that if the set defined in (20) is empty for some $t \in U\left(t_{0}\right)$ then $\alpha_{f}(t)=\infty$, so (21) is satisfied.

In the proposition below we assume that $I$ is a finite set, at least one of $a_{i}^{*}$ is not equal to zero and all $a_{i}^{*}$ do not depend on $t$. Before stating this result, let us set

$$
J:=\left\{E \subset I: \quad\left(a_{i}^{*}\right)_{i \in E} \text { are linearly independent }\right\},
$$

and

$$
f_{E}(t, x):=\max _{i \in E}\left\langle a_{i}^{*}, x\right\rangle+b_{i}(t) \text { for all } E \in J .
$$

For every $u \in X$ and $t \in T$ such that $f(t, u)=0$ Farkas lemma (for cones) tells us that

$$
\begin{aligned}
& \forall a^{*} \in X^{*}\left(\forall h \in X,\left(\left(\forall i \in I(t, u, 0),\left\langle a_{i}^{*}, h\right\rangle \leq 0\right) \Rightarrow\left\langle a^{*}, h\right\rangle \leq 0\right)\right. \\
& \quad \Rightarrow a^{*} \in\left[0,+\infty\left[\operatorname{conv}\left\{a_{i}^{*}: i \in I(t, u, 0)\right\}\right) .\right.
\end{aligned}
$$

Proposition 4.6 Suppose that $X$ is a Hilbert space, I is a finite set, at least one of $a_{i}^{*}$ is not equal to zero and all $a_{i}^{*}$ do not depend on $t$. Then for each $t \in T$ such that $K_{f}(t) \neq \emptyset$, we have

$$
\alpha_{f}(t) \geq \min _{E \in J} \min \left\{\left\|\sum_{i \in E} \lambda_{i} a_{i}^{*}\right\|: 0 \leq \lambda_{i}, \forall i \in E, \sum_{i \in E} \lambda_{i}=1\right\}>0 .
$$


Moreover, if $K_{f}(t) \neq \emptyset$ for $t$ near $t_{0}$, then

$$
\liminf _{t \rightarrow t_{0}} \alpha_{f}(t) \geq \min _{E \in J} \min \left\{\left\|\sum_{i \in E} \lambda_{i} a_{i}^{*}\right\|: 0 \leq \lambda_{i}, \forall i \in E, \sum_{i \in E} \lambda_{i}=1\right\}>0 .
$$

Proof We use the same ideas as in the proof of [13, Theorem 4.1]. For the sake of the reader convenience, we give a detailed proof of the above proposition herein. Let us observe that the following inclusion holds true

$$
\partial_{x} f_{E}(t, x) \subset \operatorname{conv}\left\{a_{i}^{*}: i \in E\right\}, \quad \forall x \in X, \forall E \in J .
$$

Let us fix $E \in J$ and set

$$
\alpha_{E}:=d\left(0, \operatorname{conv}\left\{a_{i}^{*}: i \in E\right\}\right)=\min \left\{\left\|\sum_{i \in E} \lambda_{i} a_{i}^{*}\right\|: 0 \leq \lambda_{i}, \forall i \in E, \sum_{i \in E} \lambda_{i}=1\right\} .
$$

Then, since $\left(a_{i}^{*}\right)_{i \in E}$ are linearly independent, we have

$$
d\left(0, \partial_{x} f_{E}(t, x) \geq \alpha_{E}>0, \quad \forall x \notin K_{f_{E}}(t) .\right.
$$

Observe that $\alpha_{E} \leq \alpha_{f_{E}}(t)$, where $\alpha_{f_{E}}(t)$ is definite as in (9). So using the equivalence $(8) \Longleftrightarrow(9)$, we get

$$
\alpha_{E} d\left(x, K_{f_{E}}(t)\right) \leq \max \left\{0, f_{E}(t, x)\right\}, \forall x \in X .
$$

The proof is then terminated if we show that for each $x \notin K_{f}(t)$ there exists $E \in J$ such that

$$
d\left(x, K_{f}(t)\right)=d\left(x, K_{f_{E}}(t)\right) .
$$

Indeed, let $x \notin K_{f}(t)$, then there exists $u$ in $K_{f}(t)$ such that

$$
d\left(x, K_{f}(t)\right)=\|x-u\|
$$

or equivalently

$$
\begin{aligned}
& f(t, u)=0 \\
& \quad \text { and } \forall h \in X,\left(\forall i \in I(t, u, 0),\left\langle a_{i}^{*}, h\right\rangle \leq 0 \Rightarrow\langle x-u, h\rangle \leq 0\right) .
\end{aligned}
$$

By (22), $x-u \in\left[0,+\infty\left[\operatorname{conv}\left\{a_{i}^{*}: i \in I(t, u, 0)\right\}\right.\right.$. So there exist $\lambda_{i} \geq 0, i \in I(t, u, 0)$, not all equal to zero (because $x \neq u$ ), such that

$$
x-u=\sum_{i \in I(t, u, 0)} \lambda_{i} a_{i}^{*} .
$$

If $\left(a_{i}^{*}\right)_{i \in I(t, u, 0)}$ are linearly independent then put $E^{\prime}:=I(t, u, 0) \in J$ and observe that for every $i \in E^{\prime},\left\langle a_{i}^{*}, u\right\rangle+b_{i}(t)=0$ and $\|x-u\|=d\left(x, K_{f_{E^{\prime}}}(t)\right)$. Since

$$
f_{E^{\prime}}(t, x) \leq f(t, x) \text { and } d\left(x, K_{f}(t)\right)=d\left(x, K_{f_{E^{\prime}}}(t)\right),
$$

then the result follows from (23). So suppose there exist $\mu_{i} \in R, i \in I(t, u, 0)$, not all equal to zero such that

$$
\sum_{i \in I(t, u, 0)} \mu_{i} a_{i}^{*}=0
$$

Hence for all $s \in R$

$$
\sum_{i \in I(t, u, 0)}\left(\lambda_{i}+s \mu_{i}\right) a_{i}^{*}=x-u
$$


Our problem is to find $s \geq 0$ and $i_{0} \in I(t, u, 0)$ such that $\lambda_{i_{0}}+s \mu_{i_{0}}=0$ and $\lambda_{i}+s \mu_{i} \geq 0$ for $i \neq i_{0}$. Set $I_{0}=\left\{i \in I(t, u, 0): \quad \mu_{i}<0\right\}$ and suppose that $I_{0} \neq \emptyset$. For all $i \in I_{0}$, $\lambda_{i}+\beta \mu_{i} \geq 0$ iff $\beta \leq \min _{i \in I_{0}} \frac{-\lambda_{i}}{\mu_{i}}$. So let $i_{0} \in I(t, u, 0)$ be such that $\min _{i \in I_{0}} \frac{-\lambda_{i}}{\mu_{i}}=\frac{-\lambda_{i_{0}}}{\mu_{i_{0}}}$ and put $s=\frac{-\lambda_{i_{0}}}{\mu_{i_{0}}}$. Then

$$
\lambda_{i_{0}}+s \mu_{i_{0}}=0 \text { and } \lambda_{i}+t \mu_{i} \geq 0 \forall i \neq i_{0} .
$$

By induction we show that $x-u$ is a positive combination of linearly independent family of $\left(a_{i}^{*}\right)_{i \in E}$, with $E \subset I(t, u, 0)$, or equivalently, $\|x-u\|=d\left(x, K_{f_{E}}(t)\right.$ ) (keep in mind that $\left\langle a_{i}^{*}, u\right\rangle+b_{i}(t)=0$ for every $\left.i \in I(t, u, 0)\right)$.

Taking into account that equality can be expressed as two inequalities we obtain the following corollary of Proposition 4.6.

Corollary 4.7 Let $\left\{a_{i}^{*}: i \in I\right\}$ be a finite family of vectors of a Hilbert space $X$, with at least one of $a_{i}^{*}$ not being equal to zero, and let, for each $i \in I, b_{i}: T \rightarrow \mathbb{R}$ be a function. Consider the set

$$
S(t):=\left\{x \in X:\left\langle a_{i}^{*}, x\right\rangle+b_{i}(t)=0 \forall i \in I\right\}
$$

and the function $f: T \times X \mapsto \mathbb{R}$ defined by

$$
f(t, x)=\max _{i \in I}\left|\left\langle a_{i}^{*}, x\right\rangle+b_{i}(t)\right| .
$$

If $S(t) \neq \emptyset$ for $t$ near $t_{0}$, then

$$
\liminf _{t \rightarrow t_{0}} \alpha_{f}(t)>0 .
$$

In order to deal with the set of indexes $I$ being denumerable, in fact it is enough to have that the set defined in (20) is at most countable, and to get (21) we need a more sophisticated tool. Namely, in what we do herein is employment of an approximate technique. We approximate subgradients of the convex function defined in (14) by subgradients of functions

$$
f_{n}(\cdot):=\max _{k \in\{1, \ldots, n\}}\left\langle a_{k}^{*}(t), \cdot\right\rangle+b_{k}(\cdot),
$$

which subdifferentials are possible to calculate explicitly. For this reason in the next theorem we use the Attouch theorem. The price for the use of the tool is the need to assume that the space is reflexive.

Theorem 4.8 Let $X$ be a reflexive Banach space and $t_{0} \in T$ be fixed. Assume that for a given $\delta>0$ and a neighborhood of $t_{0}$, say $U\left(t_{0}\right) \subset T$, for every $t \in U\left(t_{0}\right)$ we are able to choose a nonempty denumerable subset $I(t) \subset I$ such that

$$
y \notin K_{f}(t) \Longrightarrow\left\{\begin{array}{c}
\min \{1, f(t, y)\} \leq p(t, y) \text { if } K_{f}(t) \neq \emptyset, \\
\min \left\{\inf _{u \notin K_{f}(t)} f(t, u)+1, f(t, y)\right\} \\
\leq p(t, y) \text { if } \operatorname{dom} f(t, \cdot) \backslash K_{f}(t) \neq \emptyset
\end{array}\right.
$$

where $p(t, y):=\sup _{i \in I(t)}\left\langle a_{i}(t)^{*}, y\right\rangle+b_{i}(t)$, and

$$
\stackrel{\circ}{B}(0, \delta) \cap \bigcup_{t \in U\left(t_{0}\right)} \operatorname{conv} \bigcup_{y \notin K_{f}(t)}\left\{a_{i}^{*}(t) \mid i \in I(t),\left\langle a_{i}^{*}(t), y\right\rangle+b_{i}(t)>0\right\}=\emptyset .
$$

Then $\alpha_{f}(t) \geq \delta$ for every $t \in U\left(t_{0}\right)$, so

$$
\liminf _{t \longrightarrow t_{0}} \alpha_{f}(t) \geq \delta .
$$


Proof We show that for every $t \in U\left(t_{0}\right), y \in X \operatorname{such}_{\text {that }} \inf _{u \notin K_{f}(t)} f(t, u)+1>f(t, y)>0$ we have

$$
\partial_{x} f(t, y) \subset \mathrm{cl} \text { conv } \bigcup_{u \notin K_{f}(t)}\left\{a_{i}^{*}(t) \mid i \in I(t),\left\langle a_{i}^{*}(t), u\right\rangle+b_{i}(t)>0\right\} .
$$

For this purpose let us fix $t \in U\left(t_{0}\right)$ and $y \in X \operatorname{such}$ that $\inf _{u \notin K_{f}(t)} f(t, u)+1>f(t, y)>0$ and $\partial_{x} f(t, y) \neq \emptyset$. By the lower semi-continuity of $f(t, \cdot)$ there is $r>0$ such that $f(t, u)>0$ for every $u \in \mathbf{B}(y, r)$. Let us assume that $\left\{i_{1}, i_{2}, \ldots\right\}=I(t)$ and define a sequence of convex functions $f_{n}: X \longrightarrow \mathbb{R}$ as follows

$$
f_{n}(v):=\max _{k \in\{1, \ldots, n\}}\left\langle a_{i_{k}}^{*}(t), v\right\rangle+b_{i_{k}}(t) .
$$

For every $n \in \mathbb{N}$ and $v \in \mathbf{B}(y, r)$ we have

$$
f_{n}(v) \leq f_{n+1}(v), \quad f_{n}(v) \longrightarrow p(t, v),
$$

so $p(t, \cdot)+\psi_{\mathbf{B}(y, r)}(\cdot)$ is the Mosco limit of the sequence $\left\{f_{n}+\psi_{\mathbf{B}(y, r)}\right\}$ and Theorem 2.2 can be applied to the sequence $\left\{f_{n}+\psi_{\mathbf{B}(y, r)}\right\}$, where $\psi_{\mathbf{B}(y, r)}$ is equal to 0 on the ball and $+\infty$ outside the ball. Hence any subgradient $x^{*} \in \partial_{x} p(t, y)$ is the strong limit of subgradients of functions $f_{n}$ but for $n$ large enough we get

$$
\begin{aligned}
\partial f_{n}(y) & \subset \operatorname{cl} \operatorname{conv}\left\{a_{i_{k}}^{*}(t) \mid k \in \mathbb{N} \text { and }\left\langle a_{i_{k}}^{*}(t), y\right\rangle+b_{i_{k}}(t)>0\right\} \\
& \subset \operatorname{cl} \operatorname{conv}\left\{a_{i}^{*}(t) \mid i \in I(t) \text { and }\left\langle a_{i}^{*}(t), y\right\rangle+b_{i}(t)>0\right\} .
\end{aligned}
$$

Thus we have

$$
\partial_{x} p(t, y) \subset \mathrm{cl} \operatorname{conv}\left\{a_{i}^{*}(t) \mid i \in I(t) \text { and }\left\langle a_{i}^{*}(t), y\right\rangle+b_{i}(t)>0\right\},
$$

which by the assumptions implies

$$
d\left(0, \partial_{x} p(t, y)\right) \geq \delta .
$$

Let us fix $x^{*} \in \partial_{x} f(t, y)$. For any $z \in X$ choose $s_{z}>0$ such that $\left\langle x^{*}, s(z-y)\right\rangle+$ $f(t, y)<1+\inf _{u \notin K_{f}(t)} f(t, u)$ and $y+s(z-y) \in \mathbf{B}(y, r)$ for every $\left.\left.s \in\right] 0, s_{z}\right]$. Since $\min \left\{\inf _{u \notin K_{f}(t)} f(t, u)+1, f(t, y+s(z-y)\} \leq p(t, y+s(z-y))\right.$, so

$$
\left.\left.\left\langle x^{*}, s(z-y)\right\rangle \leq p(t, y+s(z-y))-p(t, y) \text { for every } s \in\right] 0, s_{z}\right],
$$

and hence $x^{*} \in \partial_{x} p(t, y)$, which by (26) implies

$$
d\left(0, \partial_{x} f(t, y)\right) \geq \delta .
$$

Consider the case $K_{f}(t) \neq \emptyset$ and fix $z \in \operatorname{dom} f(t, \cdot) \backslash K_{f}(t)$ (if $\operatorname{dom} f(t, \cdot) \backslash K_{f}(t)=\emptyset$ then $\alpha_{f}(t)=\infty$ and we are done). The set $K_{f}(t)$ is convex and closed, so by the reflexivity there is $x(z) \in K_{f}(t)$ such that $\|z-x(z)\|=d\left(z, K_{f}(t)\right)$. For every $\left.s \in\right] 0,1[$ we have

$$
f(t, z+s(x(z)-z)) \leq(1-s) f(t, z),
$$

and

$$
d\left(z+s(x(z)-z), K_{f}(t)\right)=(1-s) d\left(z, K_{f}(t)\right),
$$

hence

$$
\frac{f(t, z+s(x(z)-z))}{d\left(z+s(x(z)-z), K_{f}(t)\right)} \leq \frac{f(t, z)}{d\left(z, K_{f}(t)\right)}
$$


which by (7) implies

$$
\alpha_{f}(t)=\inf _{x \notin K_{f}(t), f(t, x)<1} \frac{f(t, x)}{d\left(x, K_{f}(t)\right)} .
$$

Let us assume that $\alpha_{f}(t)<\inf _{x \notin K_{f}(t), f(t, x)<1} d\left(0, \partial_{x} f(t, x)\right)$. Take any $\left.\epsilon \in\right] \alpha_{f}(t)$, $\inf _{x \notin K_{f}(t), f(t, x)<1} d\left(0, \partial_{x} f(t, x)\right)\left[\right.$ and find $v \notin K_{f}(t)$ such that $f(t, v)<1$ and $f(t, v)<$ $\epsilon d\left(v, K_{f}(t)\right)$. Choose $\left.\lambda \in\right] \frac{f(t, v)}{\epsilon}, d\left(v, K_{f}(t)\right)\left[\right.$. By Theorem 2.4, applied to $f^{+}(t, \cdot):=$ $\max \{0, f(t, \cdot)\}$, there is $w \in X$ such that

$$
\|w-v\| \leq \lambda<d\left(v, K_{f}(t)\right), \quad f(t, w) \leq f^{+}(t, v),
$$

and

$$
\partial_{x} f^{+}(t, w) \cap \mathbf{B}(0, \epsilon) \neq \varnothing,
$$

so $f(t, w) \in] 0,1\left[\right.$ (thus $f^{+}(t, w)=f(t, w)$, and $\partial_{x} f^{+}(t, w)=\partial_{x} f(t, w)$ ) and $\left\|w^{*}\right\| \leq \epsilon$ for some $w^{*} \in \partial_{x} f(t, w)$, which contradicts the choice of $\epsilon$. We conclude that in the case $K_{f}(t) \neq \emptyset$ by (27) we get $\alpha_{f}(t) \geq \delta$.

If $K_{f}(t)=\emptyset$ then $f(t, u)>0$ for every $u \in X$, so either $\operatorname{dom} f(t, \cdot)=\emptyset$ and $\alpha_{f}(t)=\infty$ or $\operatorname{dom} f(t, \cdot) \neq \varnothing$ (in the latter case $\inf _{x \in X} d\left(0, \partial_{x} f(t, x)\right)=0$ ). We exclude the latter case. For this aim fix $\left.\epsilon \in] 0, \frac{\delta}{4}\right]$. By Theorem 2.4 there is $w \in X$ such that

$$
0<f(t, w)<\inf _{u \notin K_{f}(t)} f(t, u)+1
$$

and

$$
\partial_{x} f(t, w) \cap \mathbf{B}(0, \epsilon) \neq \emptyset .
$$

so $f(t, w) \in] 0, \inf _{u \notin K_{f}(t)} f(t, u)+1\left[\right.$ and $\left\|w^{*}\right\| \leq \epsilon$ for some $w^{*} \in \partial_{x} f(t, w)$. Hence by (27) we get $\epsilon \geq \delta$ but it contradicts the choice of $\epsilon$, so it is impossible that $K_{f}(t)=\emptyset$ and $\operatorname{dom} f(t, \cdot) \neq \emptyset$. Thus $\alpha_{f}(t) \geq \delta$ for every $t \in U\left(t_{0}\right)$ whenever $K_{f}(t) \neq \emptyset$ or $K_{f}(t)=\emptyset$, which implies the statements

Let us observe that (25) is fulfilled whenever

$$
\stackrel{\circ}{B}(0, \delta) \cap \mathrm{conv} \bigcup_{y \notin K_{f}(t)}\left\{a_{i}^{*}(t) \mid i \in I,\left\langle a_{i}^{*}(t), y\right\rangle+b_{i}(t)>0\right\}=\emptyset,
$$

see also Example 4.12. Of course having $I$ denumerable we see that it is easy to check that implication (24) is satisfied (for example putting $I(t):=I$ for all $t \in T$, see Theorem 4.11), so assumptions of Theorem 4.8 are not difficult to be verified in this case. Whenever $X$ is assumed to be separable and the family $\left\{a_{i}^{*}(t) \mid i \in I\right\}$ is bounded for every $t \in T$, then implication (24) is also valid, even when $I$ is not denumerable, it is discussed in the Remark below.

Remark 4.9 Let $X$ be a separable Banach space and the family $\left\{a_{i}^{*}(t) \mid i \in I\right\}$ be bounded for every $t \in T$. For every $t \in T$ for which $\operatorname{dom} f(t, \cdot) \neq \emptyset$ let us chose a denumerable subset $D(t) \subset \operatorname{dom} f(t, \cdot)$ such that $\operatorname{dom} f(t, \cdot) \subset \operatorname{cl} D(t)$ and for every $y \in \operatorname{dom} f(t, \cdot)$ there is a sequence $\left\{y_{n}\right\}_{n \in \mathbb{N}}^{\infty} \subset D(t)$ with $y_{n} \longrightarrow y, f\left(t, y_{n}\right) \longrightarrow f(t, y)$ ( the choice of $D(t)$ could be carried out as follows: take a countable dense subset of $\{(y, \alpha) \in X \times \mathbb{R}: \alpha \geq f(t, y)\}$, say $E(t)$, and put $D(t):=\{y \in X: \exists \alpha \in \mathbb{R},(y, \alpha) \in E(t)\})$. For every $y \in D(t)$ let us choose a denumerable subset $I(t, y) \subset I$ such that

$$
\sup _{i \in I(t, y)}\left\langle a_{i}^{*}(t), y\right\rangle+b_{i}(t)=f(t, y)
$$


and put

$$
I(t):=\bigcup_{y \in D(t)} I(t, y)
$$

Let us observe that for the set $I(t)$ implication (24) holds true whenever $\operatorname{dom} f(t, \cdot) \neq \emptyset$. In fact, we have $f(t, u) \geq p(t, u)$ for every $u \in X$. Take $u \in X,\left\{y_{n}\right\}_{n \in \mathbb{N}}^{\infty} \subset D(t)$ such that $y_{n} \longrightarrow u, f\left(t, y_{n}\right) \longrightarrow f(t, u)$. Observe that

$$
p(t, u)-p\left(t, y_{n}\right) \geq \inf _{i \in I}\left\langle a_{i}^{*}(t), u-y_{n}\right\rangle \longrightarrow 0,
$$

so $p(t, u) \geq \lim _{n \longrightarrow \infty} f\left(t, y_{n}\right)=f(t, u)$, thus $p(t, u)=f(t, u)$. Let us also observe that the boundedness of the family $\left\{a_{i}^{*}(t) \mid i \in I\right\}$ and non-emptiness of the domain $\operatorname{domf}(t, \cdot)$ implies $\operatorname{domf}(t, \cdot)=X$.

The arguments used in the proof of Theorem 4.8 allows us to extend Proposition 4.5 from the finite case to the denumerable one in the reflexive Banach setting.

Theorem 4.10 Let $X$ be a reflexive Banach space and $t_{0} \in T$ be fixed. Assume for some given $\epsilon>0$ and a neighborhood of $t_{0}$, say $U\left(t_{0}\right) \subset T$, the set

$$
\bigcup_{y \notin K_{f}(t)} I(t, y, \epsilon)
$$

is nonempty and denumerable and for every $t \in U\left(t_{0}\right), \operatorname{dom} f(t, \cdot)$ is closed and for some $\delta>0$

$$
\inf _{\substack{t \in U\left(t_{0}\right) \\ y \notin K_{f}^{(t)}}} d\left(0,\left\{\operatorname{conv}\left\{a_{i}^{*}(t) \mid i \in I(t, y, \epsilon)\right\}+N(\operatorname{dom} f(t, \cdot), y)\right\}\right) \geq \delta .
$$

Then

$$
\alpha_{f}(t) \geq \delta \forall t \in U\left(t_{0}\right)
$$

Proof Put $D(t)=\operatorname{dom} f(t, \cdot)$ and fix $t \in U\left(t_{0}\right)$ and $y \in X$ such that $\infty>f(t, y)>0$ and $\partial_{x} f(t, y) \neq \varnothing$. By the lower semi-continuity of $f(t, \cdot)$ there is $r>0$ such that $f(t, u)>0$ for every $u \in \mathbf{B}(y, r)$. Let $\left.\varepsilon^{\prime} \in\right] 0, \min (\varepsilon, r)\left[\right.$. Let us assume that $\left\{i_{1}, i_{2}, \ldots\right\}=$ $\bigcup_{u \in \mathbf{B}(y, r)} I\left(t, u, \epsilon^{\prime}\right)$ and define a sequence of convex functions $f_{n}: X \longrightarrow \mathbb{R}$ as follows

$$
f_{n}(v):=\max _{k \in\{1, \ldots, n\}}\left\langle a_{i_{k}}^{*}(t), v\right\rangle+b_{i_{k}}(t) .
$$

For every $n \in \mathbb{N}$ and $v \in \mathbf{B}(y, r)$ we have

$$
f_{n}(v) \leq f_{n+1}(v) .
$$

Then $\left(f_{n}\right)$ converges to some function $\tilde{f}_{\mathcal{E}^{\prime}}$ on $\mathbf{B}(y, r)$ such that $\tilde{f}_{\mathcal{E}^{\prime}}(\cdot)=f(t, \cdot)$ on $D(t) \cap$ $\mathbf{B}(y, r)$. So $\tilde{f}_{\mathcal{E}^{\prime}}(\cdot)+\psi_{\mathbf{B}(y, r) \cap D(t)}(\cdot)$ is the Mosco limit of the sequence $\left\{f_{n}+\psi_{\mathbf{B}(y, r) \cap D(t)}\right\}$ (the functions are convex and lower semi-continuous) and Theorem 2.2 can be applied to the sequence $\left\{f_{n}+\psi_{\mathbf{B}(y, r) \cap D(t)}\right\}$, where $\psi_{C}$ is equal to 0 on the set $C$ and $+\infty$ outside this set. Hence for any $u^{\prime} \in \stackrel{\circ}{B}(y, r) \cap D(t)$, any subgradient $\left.x^{*} \in \partial\left[\tilde{f}_{\mathcal{E}^{\prime}}+\psi_{D(t)}\right\}\right]\left(u^{\prime}\right)$ is a strong limit of subgradients $\left(x_{n}^{*}\right)$, with $x_{n}^{*} \in \partial f_{n}\left(u^{\prime}\right)+N\left(D(t), u^{\prime}\right)$ for all $n$, but

$$
\partial f_{n}\left(u^{\prime}\right)+N\left(D(t), u^{\prime}\right) \subset \operatorname{cl} \operatorname{conv}\left\{a_{i_{k}}^{*}(t) \mid k \in \mathbb{N}\right\}+N\left(D(t), u^{\prime}\right) .
$$


The assumptions of the theorem and the last inclusion ensure that

$$
\left\|x_{n}^{*}\right\| \geq \delta \text {. }
$$

As $\left(x_{n}^{*}\right)$ strongly converges to $x^{*}$, we have

$$
\left\|x^{*}\right\| \geq \delta
$$

and hence

$$
\left.d\left(0, \partial\left[\tilde{f}_{\varepsilon^{\prime}}+\psi_{D(t)}\right\}\right]\left(u^{\prime}\right)\right) \geq \delta .
$$

Now, pick $x^{*} \in \partial_{x} f(t, y)$ or equivalently

$$
f(t, y)-\left\langle x^{*}, y\right\rangle \leq f(t, u)-\left\langle x^{*}, u\right\rangle \forall u \in X .
$$

Since for all $u \in \mathbf{B}(y, r) \cap D(t), \emptyset \neq I\left(t, u, \varepsilon^{\prime}\right) \subset\left\{i_{1}, i_{2}, \ldots\right\}$, we obtain

$$
\tilde{f}_{\varepsilon^{\prime}}(u)=f(t, u),
$$

so

$$
\tilde{f}_{\varepsilon^{\prime}}(y)-\left\langle x^{*}, y\right\rangle \leq \tilde{f}_{\varepsilon^{\prime}}(u)-\left\langle x^{*}, u\right\rangle \forall u \in \mathbf{B}(y, r) \cap D(t) .
$$

Thus,

$$
x^{*} \in \partial\left[\tilde{f}_{\varepsilon^{\prime}}+\psi_{D(t)}\right](y) .
$$

Combining this relation with (28), it follows that

$$
\left\|x^{*}\right\| \geq \delta
$$

and hence

$$
d\left(0, \partial_{x} f(t, y)\right) \geq \delta .
$$

It follows that

$$
\alpha_{f}(t) \geq \delta
$$

In the discrete case, i.e. $I=\mathbf{N}$, the assumptions of Theorem 4.10 can be relaxed.

Theorem 4.11 Let $X$ be a reflexive Banach space and $t_{0} \in T$ be fixed. Assume $I=\mathbb{N}$ and for some given neighborhood of $t_{0}$, say $U\left(t_{0}\right) \subset T$, and for some $\delta>0$ the following inequality holds

$$
\inf _{\substack{t \in U\left(t_{0}\right) \\ y \notin K}} d\left(0, \operatorname{conv}\left\{a_{i}^{*}(t) \mid i \in \mathbb{N}\right\}\right) \geq \delta .
$$

Then

$$
\alpha_{f}(t) \geq \delta \forall t \in U\left(t_{0}\right) .
$$

Proof The proof is similar to the previous one by considering the sequence $\left(f_{n}\right)$, where

$$
f_{n}(v)=\max _{k \in\{1, \ldots, n\}}\left\langle a_{k}^{*}(t), v\right\rangle+b_{k}(t),
$$

which converges to $f(t, \cdot)$. 
Let us also notice that the condition

$$
\stackrel{\circ}{B}(0, \delta) \cap \bigcup_{t \in U\left(t_{0}\right)} \text { conv } \bigcup_{y \notin K_{f}(t)}\left\{a_{i}^{*}(t) \mid i \in I(t),\left\langle a_{i}^{*}(t), y\right\rangle+b_{i}(t)>0\right\}=\emptyset,
$$

is equivalent to

$$
\stackrel{\circ}{B}(0, \delta) \cap \bigcup_{t \in U\left(t_{0}\right)} \mathrm{cl} \text { conv } \bigcup_{y \notin K_{f}(t)}\left\{a_{i}^{*}(t) \mid i \in I(t),\left\langle a_{i}^{*}(t), y\right\rangle+b_{i}(t)>0\right\}=\emptyset,
$$

which in the reflexive Banach spaces is the same as

$$
\stackrel{\circ}{B}(0, \delta) \cap \bigcup_{t \in U\left(t_{0}\right)} \mathrm{cl}^{*} \operatorname{conv} \bigcup_{y \notin K_{f}(t)}\left\{a_{i}^{*}(t) \mid i \in I(t),\left\langle a_{i}^{*}(t), y\right\rangle+b_{i}(t)>0\right\}=\emptyset .
$$

In Theorem 4.8 we use (29) in order to guarantee the positiveness of lower limit of error bounds. In a nonreflexive Banach space (30) implies (29) but the reverse is not always true. Of course we could use (30) in Theorem 4.8 instead of (29) and the result would be the same. However in nonreflexive Banach spaces it is not possible. In the next theorem we propose a result where (30) is used instead of (29) and the space is assumed to be weakly compactly generated. However as a price for that we have to assume the family of functions $f(t, \cdot), t \in T$ consists of continuous functions. The continuity assumptions can be relaxed using a technical condition from [27], for the sake of simplicity we do not do it- the interested reader can do it repeating the ideas. Let us start with an example illuminating (30).

Example 4.12 Let $X$ be a Banach space and $A \subset X^{*}$ be a convex weak* closed subset such that $0 \notin A$, for example $A:=\mathbf{B}\left(z^{*}, r\right)$, where $\left\|z^{*}\right\|>r, z^{*} \in X^{*}$. Assume that for every $t \in U\left(t_{0}\right)$ and $y \in X$ we have

$$
\left\{a_{i}^{*}(t) \mid i \in I,\left\langle a_{i}^{*}(t), y\right\rangle+b_{i}(t)>0\right\} \subset A,
$$

then (30) and (25) are satisfied for $\delta>0$ sufficiently small.

In the proof of the theorem below we need the property that whenever the interior of the domain is nonempty, i.e int $\operatorname{dom} f(t, \cdot) \neq \emptyset$ then the Hoffman constant can be calculated in the interior of the domain, namely we have

Proposition 4.13 For every $t \in T$ such that int $\operatorname{dom} f(t, \cdot) \neq \emptyset$ we have

$$
\alpha_{f}(t)=\inf _{y \in \text { int } \operatorname{dom} f(t, \cdot) \backslash K_{f}(t)} d\left(0, \partial_{x} f(t, y)\right) .
$$

Proof Assume that (31) does not hold, i.e.

$$
\alpha_{f}(t)<\inf _{y \in \operatorname{int} \operatorname{dom} f(t, \cdot) \backslash K_{f}(t)} d\left(0, \partial_{x} f(t, y)\right) .
$$

Fix any $\left.z \in \operatorname{dom} f(t, \cdot) \backslash K_{f}(t), \lambda \in\right] 0, d\left(z, K_{f}(t)\right)[$ and $\epsilon>0$ such that

$$
\begin{aligned}
\alpha_{f}(t) & \leq \frac{f(t, z)}{d\left(z, K_{f}(t)\right)}<\frac{f(t, z)}{\lambda}<\epsilon \\
& <\inf _{y \in \operatorname{int} \operatorname{dom} f(t, \cdot) \backslash K_{f}(t)} d\left(0, \partial_{x} f(t, y)\right) .
\end{aligned}
$$

Now let us apply Theorem 2.4 for the function $p(\cdot):=\max \{0, f(t, \cdot)\}$. There is $u \in X$ such that $\|u-z\| \leq \lambda<d\left(z, K_{f}(t)\right)$ and $\partial p(u) \cap \mathbf{B}(0, \epsilon) \neq \emptyset$. Hence $p(u)>0$ and $\left.\partial p(u)=\partial_{x} f(t, u)\right)$, so $\left.\partial_{x} f(t, u)\right) \cap \mathbf{B}(0, \epsilon) \neq \emptyset$, which contradicts the last inequality in (32). 
Theorem 4.14 Let $X$ be a weakly compactly generated Banach space and $t_{0} \in T$ be fixed. Assume that for some $\epsilon>0$ and a neighborhood of $t_{0}$, say $U\left(t_{0}\right) \subset T$, the sets

$$
\text { int } \operatorname{dom} f(t, \cdot)
$$

are nonempty for every $t \in U\left(t_{0}\right)$ and the set

$$
\bigcup_{y \notin K_{f}(t)} I(t, y, \epsilon)
$$

is nonempty and denumerable, and for some $\delta>0$

$$
\stackrel{\circ}{B}(0, \delta) \cap \bigcup_{t \in U\left(t_{0}\right)} c l^{*} \operatorname{conv}\left\{a_{i}^{*}(t) \mid i \in I(t, y, \epsilon), f(t, y)>0\right\}=\emptyset .
$$

Then $\alpha_{f}(t) \geq \delta$ for every $t \in U\left(t_{0}\right)$, thus

$$
\liminf _{t \rightarrow t_{0}} \alpha_{f}(t) \geq \delta .
$$

Proof It follows from Proposition 4.13 that it is enough to show that for every $t \in U\left(t_{0}\right), y \in$ int dom $f(t, \cdot) \backslash K_{f}(t)$ we have

$$
\partial_{x} f(t, y) \subset \mathrm{cl}^{*} \operatorname{conv}\left\{a_{i}^{*}(t) \mid i \in I(t, u, \epsilon), f(t, u)>0\right\} .
$$

For this reason let us fix $t \in U\left(t_{0}\right)$ and $y \in X$ such that $y \in \operatorname{int} \operatorname{dom} f(t, \cdot) \backslash K_{f}(t)$ (of course $\left.\partial_{x} f(t, y) \neq \emptyset\right)$. By the lower semi-continuity of $f(t, \cdot)$ there is $r>0$ such that $\mathbf{B}(y, r) \subset$ int $\operatorname{dom} f(t, \cdot) \backslash K_{f}(t)$ and $f(t, \cdot)$ is bounded from the above on $\mathbf{B}(y, r)$. Let us assume that $\left\{i_{1}, i_{2}, \ldots\right\}=\bigcup_{u \in \mathbf{B}(y, r)} I(t, u, \epsilon)$ and define a sequence of convex functions $f_{n}: X \longrightarrow \mathbb{R}$ as follows

$$
f_{n}(v):=\max _{k \in\{1, \ldots, n\}}\left\langle a_{i_{k}}^{*}(t), v\right\rangle+b_{i_{k}}(t) .
$$

For every $n \in \mathbb{N}$ and $v \in \mathbf{B}(y, r)$ we have

$$
f_{n}(v) \leq f_{n+1}(v), \quad f_{n}(v) \longrightarrow f(t, v),
$$

so $f(t, \cdot)+\psi_{\mathbf{B}(y, r)}(\cdot)$ is the Mosco limit of the sequence $\left\{f_{n}+\psi_{\mathbf{B}(y, r)}\right\}$, where $\psi_{\mathbf{B}(y, r)}$ is equal to 0 on the ball and $+\infty$ outside the ball and Theorem 2.3 can be applied (keep in mind that by the choice of $y$ and the assumption int $\operatorname{dom} f(t, \cdot) \neq \emptyset$ we have int $\operatorname{dom} f(t, \cdot)+$ $\psi_{\mathbf{B}(y, r)}(\cdot) \neq \emptyset$, which implies that the sequence $\left\{f_{n}+\psi_{\mathbf{B}(y, r)}\right\}$ is uniformly bounded from the above). Hence any subgradient $x^{*} \in \partial_{x} f(t, y)$ is the weak* limit of a sequence of subgradients of $f_{n}$ 's but

$$
\begin{aligned}
& \bigcup_{n \in \mathbb{N}} \partial f_{n}(y) \subset \mathrm{cl}^{*} \operatorname{conv}\left\{a_{i_{k}}^{*}(t) \mid k \in \mathbb{N}\right\} \\
& \quad=\operatorname{cl}^{*} \operatorname{conv}\left\{a_{i}^{*}(t) \mid i \in I(t, u, \epsilon), f(t, u)>0\right\} .
\end{aligned}
$$

Thus we have

$$
\partial_{x} f(t, y) \subset \mathrm{cl}^{*} \operatorname{conv}\left\{a_{i}^{*}(t) \mid i \in I(t, u, \epsilon), f(t, u)>0\right\},
$$

which by the assumptions implies

$$
d\left(0, \partial_{x} f(t, y)\right) \geq \delta \quad \text { for every } t \in U\left(t_{0}\right), y \in X \text { with } \infty>f(t, y)>0,
$$


hence it follows that

$$
\liminf _{t \rightarrow t_{0}} \alpha_{f}(t) \geq \delta
$$

Let us point out that in the proof of the above theorem we need only the assumption that for every $y \notin K_{f}(t)$ such that $f(t, y)<\infty$ there is $\mu>0$ such that the set

$$
\bigcup_{u \notin K_{f}(t), u \in B(y, \mu)} I(t, u, \epsilon)
$$

is nonempty and denumerable.

\section{Lipschitz-like and lower semi-continuity properties of the admissible sets}

In this section we show that if

$$
\liminf _{t \longrightarrow t_{0}} \alpha_{f}(t)>0
$$

then the mapping of admissible sets of solutions $K_{f}(\cdot)$ is lower semi-continuous at $t_{0}$.

Theorem 5.1 Let $X$ be a real Banach space, $T$ be a metric space, $t_{0} \in T$ and its neighborhood $U\left(t_{0}\right) \subset T$ be given, $f: T \times X \longrightarrow \mathbb{R} \cup\{+\infty\}$ be such that

$$
K_{f}(t):=\{x \in X \mid f(t, x) \leq 0\}
$$

is nonempty at $t_{0}$, and for every $t \in T$ the function $f(t, \cdot)$ is proper convex lower semicontinuous and for every $x \in K_{f}\left(t_{0}\right)$, and for every sequence $\left\{t_{n}\right\}_{n \in \mathbb{N}} \subset T$ converging to $t_{0}$ there is a sequence, $\left\{x_{n}\right\}_{n \in \mathbb{N}} \subset X$ converging to $x$ such that

$$
\limsup _{n \rightarrow \infty} f\left(t_{n}, x_{n}\right) \leq 0 \text {. }
$$

Then, the following inequality

$$
\liminf _{t \rightarrow t_{0}} \alpha_{f}(t)>0
$$

entails the lower semi-continuity of $K_{f}(\cdot)$ at $t_{0}$.

Proof Assume that liminf $\operatorname{int}_{t \rightarrow t_{0}} \alpha_{f}(t)>0$. Let us fix $x_{0} \in K_{f}\left(t_{0}\right)$, a sequence $\left\{t_{n}\right\}_{n \in \mathbb{N}} \subset T$ converging to $t_{0}$ and a sequence, $\left\{x_{n}\right\}_{n \in \mathbb{N}} \subset X$ converging to $x_{0}$ such that

$$
\limsup _{n \rightarrow \infty} f\left(t_{n}, x_{n}\right) \leq 0 .
$$

First let us observe that if $K_{f}\left(t_{n}\right)=\emptyset$, then the proper convex lower-semi-continuous function $f\left(t_{n}, \cdot\right)$ is bounded from below by 0 . Thus the Ekeland Variationl Principle, see Theorem 2.4, ensures the existence of a pair $\left(z_{n}, z_{n}^{*}\right) \in \partial_{x} f\left(t_{n}, \cdot\right)$ such that

$$
\alpha_{f}\left(t_{n}\right) \leq\left\|z_{n}^{*}\right\| \leq 2^{-1} \liminf _{t \rightarrow t_{0}} \alpha_{f}(t),
$$

but it would be a contradiction for $n$ large enough, so for $n$ 's large the sets $K_{f}\left(t_{n}\right)$ are nonempty. Since the sequence $\left\{x_{n}\right\}_{n \in \mathbb{N}} \subset X$ converges to $x_{0}$, the case $x_{n} \in K_{f}\left(t_{n}\right)$ for every $n$ implies the statement immediately. So let us consider the case whenever infinite many of 
$x_{n}$ 's are out of $K_{f}\left(t_{n}\right)$. Without loss of the generality we may assume that for every $n$ large enough $x_{n} \notin K_{f}\left(t_{n}\right)$. Using equivalence (8) we get

$$
0=\lim _{n \longrightarrow \infty} \alpha_{f}\left(t_{n}\right) d\left(x_{n}, K_{f}\left(t_{n}\right)\right) \leq \limsup _{n \longrightarrow \infty} f\left(t_{n}, x_{n}\right)=0,
$$

which implies the existence of a sequence $\left\{x_{n}^{\prime}\right\}_{n \in \mathbb{N}} \subset X$ such that $x_{n}^{\prime} \longrightarrow x_{0}$ and $x_{n}^{\prime} \in K_{f}\left(t_{n}\right)$ for every $n$ large enough, thus the lower semi-continuity is proved.

In several cases (33) is a simple consequence of imposed assumptions on the involved functions. For example, let us point out that if we assume that $f$ is continuous on $T \times X$, for example assuming that $I$ is finite and $a_{i}^{*}(\cdot), b_{i}(\cdot)$ are continuous, we get (33) with $x_{n}:=x_{0}$ for every $n \in \mathbb{N}$. Condition (33) is also fulfilled with $x_{n}:=x_{0}$ for every $n \in \mathbb{N}$, whenever $f(\cdot, x)$ is upper semi-continuous for every $x \in K_{f}\left(t_{0}\right)$. If for every $\left\{t_{n}\right\}_{n \in \mathbb{N}} \subset T$ converging to $t_{0}$ we have $f\left(t_{0}, \cdot\right)=$ Mosco $-\lim _{n \longrightarrow \infty} f\left(t_{n}, \cdot\right)$, then (33) is satisfied too. Thus we see that (33) can be entangled in other assumptions and in several cases we get it immediately.

Let $f: T \times X \mapsto \mathbf{R}$ be of the form

$$
f(t, x)=\sup _{i \in I}\left\langle a_{i}^{*}(t), x\right\rangle+b_{i}(t)
$$

where $I$ is a denumerable set. We endow $T$ with a metric denoted by $d_{T}$.

Our aim here is to show how to use the positiveness of the lower limit of the Hoffman constant to get a kind of Lipschitz-like property of the admissible set defined by the function $f$.

\section{Theorem 5.2 Let $t_{0} \in T$. Suppose that}

(i) there exist $\gamma>0$ and a neighbourhood $U\left(t_{0}\right)$ of $t_{0}$ such that

$$
\left\|a_{i}^{*}(t)-a_{i}^{*}\left(t^{\prime}\right)\right\| \leq \gamma d_{T}\left(t, t^{\prime}\right),\left|b_{i}(t)-b_{i}\left(t^{\prime}\right)\right| \leq \gamma d_{T}\left(t, t^{\prime}\right), \forall i \in I, \forall t, t^{\prime} \in U\left(t_{0}\right)
$$

(ii) there exists $a>0$ such that

$$
\liminf _{t \rightarrow t_{0}} \alpha_{f}(t)>\frac{1}{a} .
$$

Then there exists a neighborhood $U_{0} \subset U\left(t_{0}\right)$ of $t_{0}$ such that

$$
K_{f}\left(t^{\prime}\right) \cap \mathbf{B}(0, r) \subset K_{f}(t)+a(r+1) \gamma d_{T}\left(t, t^{\prime}\right) \mathbf{B}(0,1), \forall t, t^{\prime} \in U_{0}, \forall r>0 .
$$

Proof By (ii), there exists a neighborhood $U_{0} \subset U\left(t_{0}\right)$ of $t_{0}$ such that

$$
\alpha_{f}(t) \geq \frac{1}{a}, \forall t \in U_{0}
$$

or equivalently

$$
d\left(x, K_{f}(t)\right) \leq a f(t, x), \forall x \notin K_{f}(t) .
$$

Let $r>0$ be arbitrary, $t, t^{\prime} \in U_{0}$ and $x \in K_{f}\left(t^{\prime}\right) \cap \mathbf{B}(0, r)$. If $x \in K_{f}(t)$, then we are done. Otherwise, relation (34) together with $i$ ) implies

$$
d\left(x, K_{f}(t)\right) \leq a\left[f(t, x)-f\left(t^{\prime}, x\right)\right] \leq a(r+1) \gamma d_{T}\left(t, t^{\prime}\right)
$$

which completes the proof.

Let us observe that whenever $K_{f}(t)$ is uniformly bounded near $t_{0}$, the above theorem asserts that $K_{f}$ is Lipschitz continuous at $t_{0}$. 
Open Access This article is distributed under the terms of the Creative Commons Attribution Noncommercial License which permits any noncommercial use, distribution, and reproduction in any medium, provided the original author(s) and source are credited.

\section{References}

1. Attouch, H.: Variational Convergence for Functions and Operators. Pitman Advanced Publishing Program, Boston (1984)

2. Attouch, H., Beer, G.: On the convergence of subdifferentials of convex functions. Arch. Math. 60, 389-400 (1993)

3. Azé, D.: A unified theory for metric regularity of multifunctions. J. Convex Anal. 13, 225-252 (2006)

4. Azé, D., Corvelec, J.-N.: On the sensitivity analysis of Hoffman constants for systems of linear inequalities. SIAM J. Optim. 12(4), 913-927 (2002)

5. Bank, B., Guddat, J., Klatte, D., Kummer, B., Tammer, K.: Non-Linear Parametric Optimization. Akademie-Verlag, Berlin (1982)

6. Bosch, P., Henrion, R., Jourani, A.: Error bounds and applications. Appl. Math. Optim. 50, 161-181 (2004)

7. Cornejo, O., Jourani, A., Zalinescu, C.: Conditioning and upper-Lipschitz inverse subdifferentials in nonsmooth optimization problems. J. Optim. Theory Appl. 95(1), 127-148 (1997)

8. Deville, R., Godefroy, G., Zizler, V.: Smoothness and Renormings in Banach Spaces. Longman Scientific and Technical, Essex (1993)

9. Ekeland, I.: Nonconvex minimization problems. Bull. Am. Math. Soc. 1, 443-474 (1979)

10. Hoffman, A.J.: On approximate solutions of systems of linear inequalities. J. Res. Natl. Bureau Stand. 49, 263-265 (1952)

11. Hogan, W.H.: Point-to-set maps in mathematical programming. SIAM Rev. 15, 591-603 (1973)

12. Holmes, R.: Geometric Functional Analysis and its Applications. Springer, New York (1975)

13. Jourani, A.: Hoffman's error bound, local controllability and sensitivity analysis. SIAM J. Control Optim. 38, 947-970 (Erratum in the same Journal) (2000)

14. Klatte, D., Thiere, G.: Error bounds for solutions of linear equations and inequalities. ZOR Math. Methods Oper. Res. 41, 191-214 (1995)

15. Li, W.: The sharp Lipschitz constants for feasible and optimal solutions of a perturbed linear program. Linear Algebra Appl. 187, 15-40 (1993)

16. Mangasarian, O.L.: A condition number of linear inequalities and equalities. Methods Oper. Res. 43, 3-15 (1981)

17. Mangasarian, O.L., Shiau, T.H.: Lipschitz continuity of solutions of linear inequalities, programs and complementarity problems. SIAM J. Control Optim. 25, 583-595 (1987)

18. Meyer, R.R.: The validity of a family of optmization methods. SIAM J. Control 8, 41-54 (1970)

19. Mohebi, H., Naraghirad, E.: Closed convex sets and their best simultaneous approximation properties with applications. Optim. Lett. 1(4), 313-328 (2007)

20. Mordukhovich, B.S.: Variational Analysis and Generalized Differentiation. I: Basic Theory. Grundlehren Series (Fundamental Principles of Mathematical Sciences). Vol. 330, Springer, Berlin (2006) (584 p)

21. Mordukhovich, B.S.: Variational Analysis and Generalized Differentiation. II: Applications. Grundlehren Series (Fundamental Principles of Mathematical Sciences). Vol. 331, Springer, Berlin (2006) (592 p)

22. Phelps, R.R.: Convex Functions, Momnotone Operators and Differentiability. 2nd edn. Springer, Berlin (1993)

23. Robinson, S.M.: Bounds for error in the solution set of a perturbed linear program. Linear Algebra Appl. 6, 69-81 (1973)

24. Tardella, F.: Existence and sum decomposition of vertex polyhedral convex envelopes. Optim. Lett. 2(3), 363-375 (2008)

25. Zagrodny, D.: On the weak* convergence of subdifferentials of convex functions. J. Conv. Anal. 12, 213-219 (2005)

26. Zagrodny, D.: Minimizers of the limit of Mosco converging functions. Arch. Math. 85(5), 440-445 (2005)

27. Zagrodny, D.: A weak* approximation of subgradient of convex function. Control Cybern. 36(3), 793-802 (2007)

28. Zălinescu, C.: Sharp estimates for Hoffman's constant for systems of linear inequalities and equalities. SIAM J. Optim. 14, 517-533 (2003)

29. Zheng, X.-Y., Ng, K.-F.: Hoffman's least error bounds for systems of linear inequalities. J. Glob. Optim. 30(4), 391-403 (2004) 